\title{
Variation of Donor and Acceptor in D-A- $\pi$-A based Cyanopyridine Dyes and its effect on Dye Sensitized Solar Cells
}

\author{
B Hemavathi ${ }^{a}$, Jayadev $V^{b c}$., Praveen C Ramamurthy ${ }^{d}$., Ranjith Krishna Paie., Narayanan Unni \\ K. N ${ }^{b, c}$., Ahipa T.N. ${ }^{a *}$, Suraj Soman ${ }^{b, c *}$, R Geetha Balakrishna ${ }^{*}{ }^{*}$
}

${ }^{a}$ Centre for Nano and Material Sciences, Jain University, Jain Global Campus, Kanakapura, Ramanagaram, Bangalore - 562112, India.

\begin{abstract}
bPhotosciences and Photonics Section, Chemical Sciences and Technology Division, CSIR-National Institute for Interdisciplinary Science and Technology, Thiruvananthapuram 695019, Kerala, India.

cAcademy of Scientific and Innovative Research (AcSIR), New Delhi 110001, India.

dDepartment of Materials Engineering, Indian Institute of Science, Bangalore-560012, India

'Technology Mission Division, Department of Science and Technology (DST), Ministry of Science and Technology, Government of India, Technology Bhavan, New Mehrauli Road, New Delhi, 110016, India
\end{abstract}

*For correspondence: br.geetha@jainuniversity.ac.in, suraj@niist.res.in,

\section{tn.ahipa@jainuniversity.ac.in}

General Methods. All chemicals and reagents were purchased from the commercial sources. The solvents were distilled before use. ${ }^{1} \mathrm{H}-\mathrm{NMR}$ and ${ }^{13} \mathrm{C}-\mathrm{NMR}$ spectra were recorded by using deuterated solvents $\left(\mathrm{CDCl}_{3}\right.$ or DMSO-d6) on a Jeol AV-III400 (L) spectrometer operating at 400 $\mathrm{MHz}$ and $100 \mathrm{MHz}$, respectively, with TMS as reference. Absorption and photoluminescence spectra were recorded with Shimadzu UV-1800 and Shimadzu RF-5301PC, respectively. Mass spectra were collected by Turbo Ion spray (ESI), SynaptG2, Waters instrument. ATR-IR spectra were recorded by using Bruker ALPHA eco-ATRIR on ZnSe crystal. Cyclic voltammetry (CV) measurements for the dyes were collected using an $\mathrm{Ag} / \mathrm{AgNO}_{3}$ as reference electrode, glassy carbon as working electrode and $\mathrm{Pt}$ wire as counter electrode in tetrabutylammonium hexafluorophosphate $\left(\mathrm{TBAPF}_{6}\right)$ as electrolyte solution $(0.1 \mathrm{M})$ in acetonitrile with CHI66OD potentiostat $\mathrm{CH}$ instrument. Theoretical calculations were done with Gaussian 09 program using B3LYP (Becke's 43 three parameters hybrid functional and Lee, Yang, and Parr's) correlational functional and 6-31G(d, p) basis set.

\section{Solar Cell Fabrication and Characterization}

The working electrodes (FTO, TEC $15 \Omega / \mathrm{Sq} \mathrm{cm}$, GreatCell Solar) were cleaned subsequently with detergent, deionized water, acetone and isopropyl alcohol, with an ultrasonic bath. Then, the cleaned working electrodes were heated to $500{ }^{\circ} \mathrm{C}$, and UV-Ozone treated for $15 \mathrm{~min}$. Later, the 
substrates were immersed into $40 \mathrm{mM} \mathrm{TiCl}_{4}$ and then kept at $70{ }^{\circ} \mathrm{C}$ for $30 \mathrm{~min}$, then washed with deionized water and ethanol subsequently. The doctor-blading technique was used to get $\mathrm{TiO}_{2}$ (GreatCell Solar) layer the substrates were then placed for a ramp heating $\left(325^{\circ} \mathrm{C}\right.$ for $15 \mathrm{~min}$, $375^{\circ} \mathrm{C}$ for $15 \mathrm{~min}, 450{ }^{\circ} \mathrm{C}$ for $15 \mathrm{~min}$ and $500^{\circ} \mathrm{C}$ for $30 \mathrm{~min}$ ). Then it was allowed to cool down to room temperature. The coated $\mathrm{TiO}_{2}$ electrodes were soaked for 15 hours in respective dye solutions $(0.3 \mathrm{mM})$ in acetonitrile. Pt paste (GreatCell Solar) over counter electrodes was coated and annealed at $380{ }^{\circ} \mathrm{C}$ for $20 \mathrm{~min}$. $25 \mu \mathrm{m}$ thick surlyn spacer was used to assemble the electrodes and standard $I^{-} I_{3}^{-}$electrolyte was filled through drilled holes and then holes were sealed using cover glass.

The current density versus voltage $(J-V)$ curve of the fabricated DSSCs were measured under AM 1.5 irradiation $\left(100 \mathrm{mWcm}^{-2}\right.$ ) using Newport class AAA simulator (Oriel Sol3A- 94023A) at room temperature. The incident-photon-to-current conversion efficiency (IPCE) spectra was measured in DC mode (Newport, 350W Xenon lamp). Electrochemical impedance spectroscopy, charge extraction (CE) and intensity modulated photo-current spectroscopy (IMPS) measurements were carried out using electrochemical workstation (Autolab PGSTAT302N) with reference to reported methods. ${ }^{1-6}$ The photo-current response was measured in warm-white LED, from $0.1 \mathrm{~Hz}$ to $10 \mathrm{kHz}$ frequency range using a $10 \%$ DC light perturbation. 


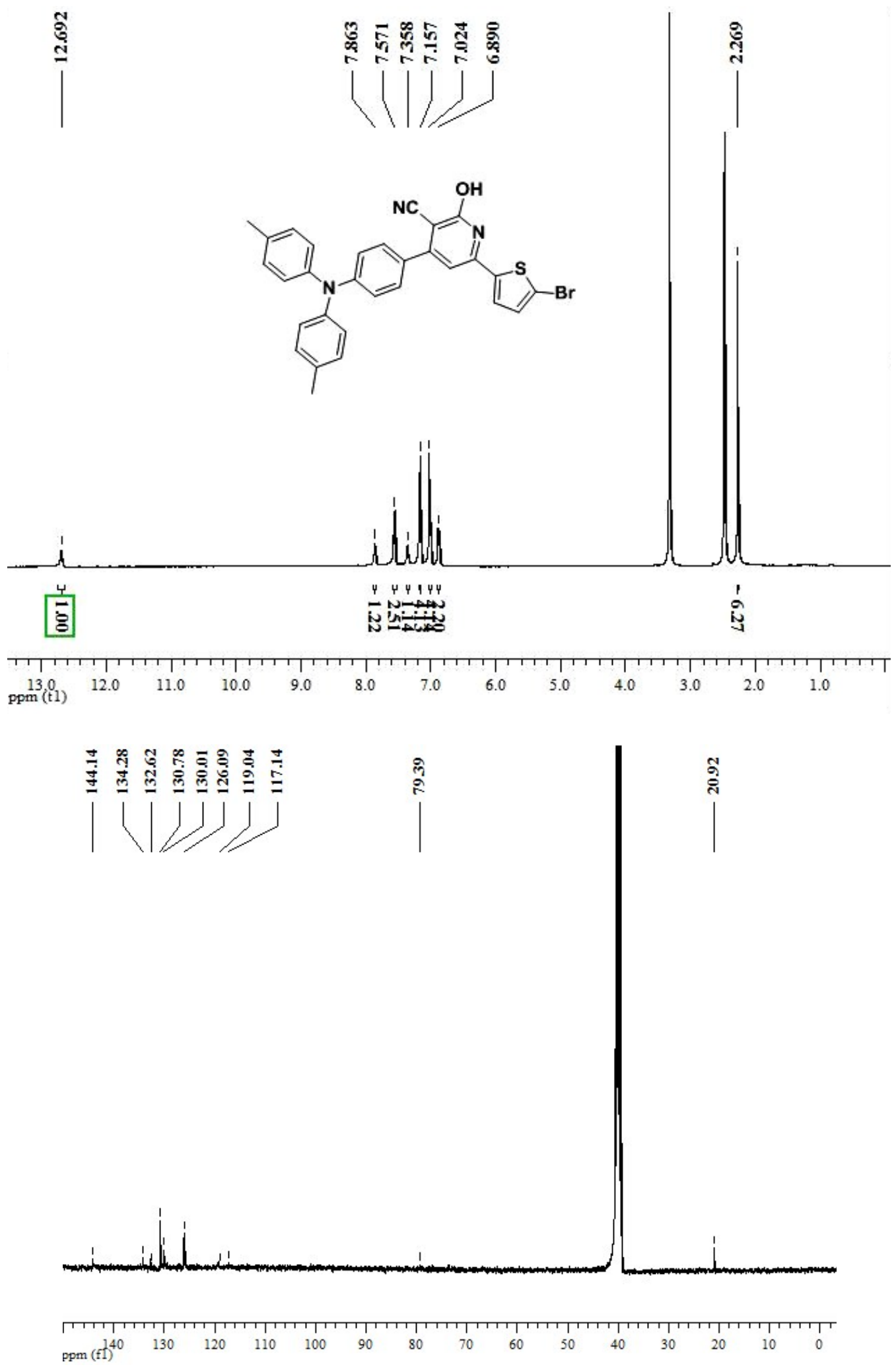

Figure S1: 1H-NMR and 13C-NMR of compound 2 

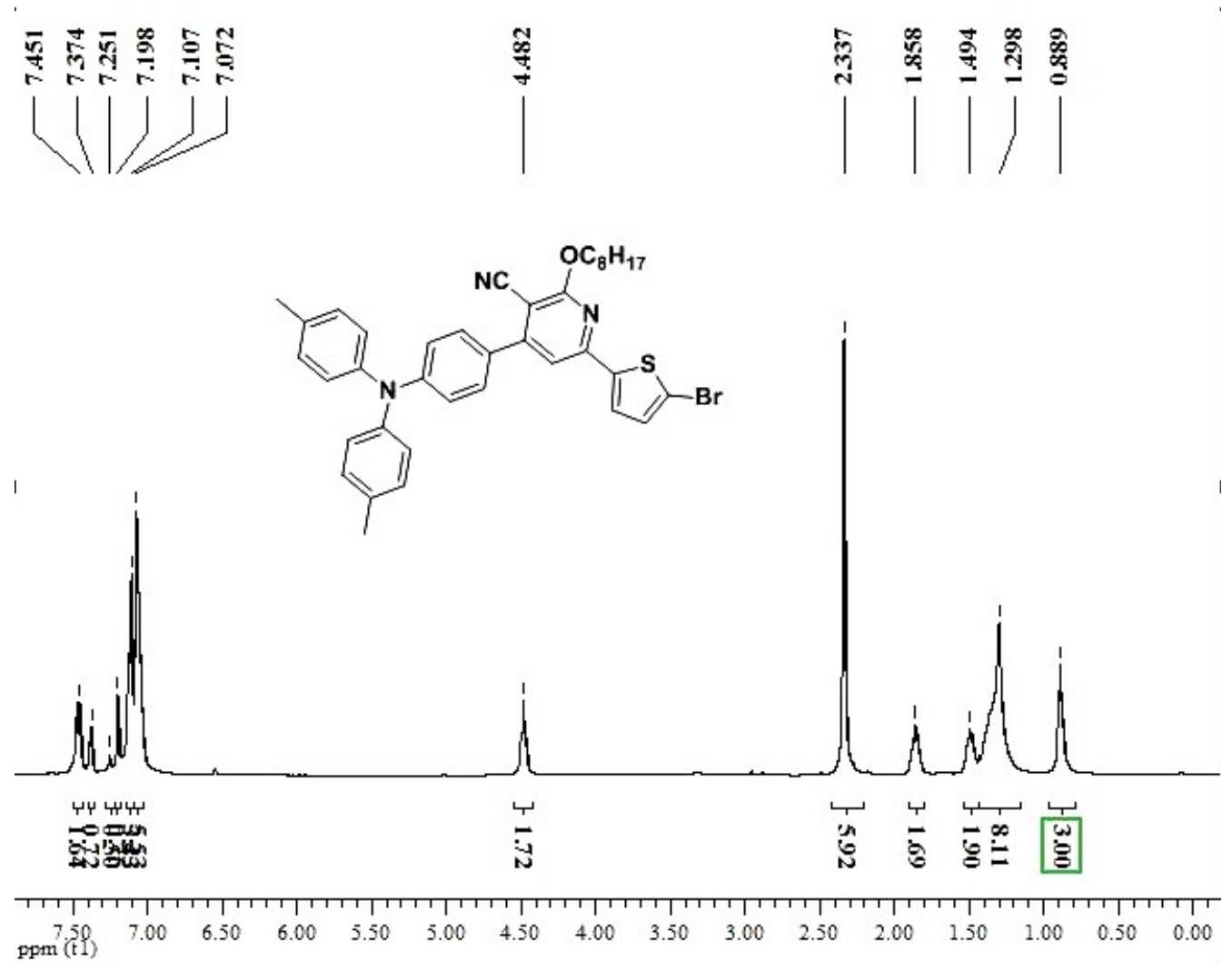

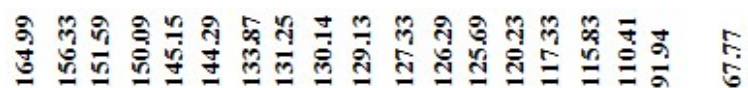

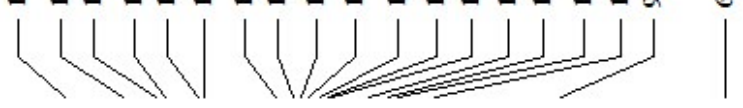

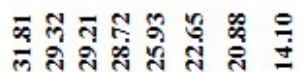
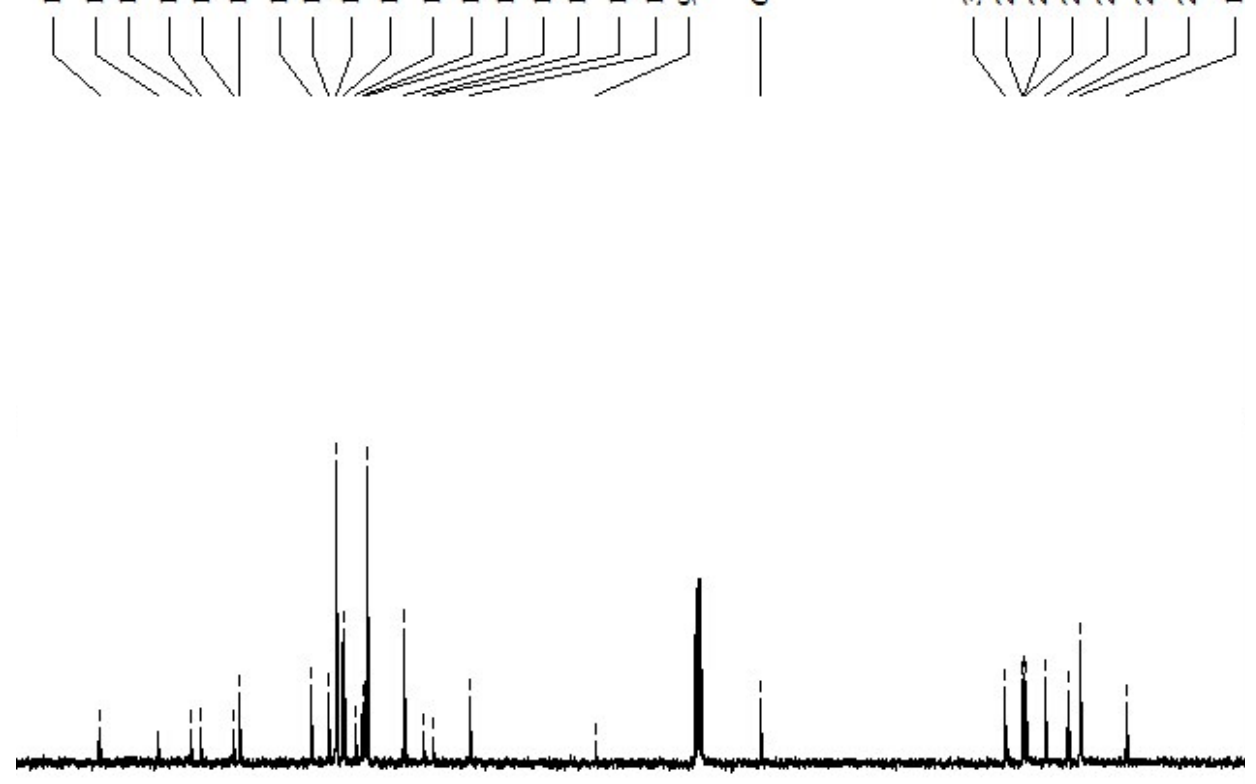

$\begin{array}{ccccccccccccccccccccc}17(f) & 160 & 150 & 140 & 130 & 120 & 110 & 100 & 90 & 80 & 70 & 60 & 50 & 40 & 30 & 20 & 10 & 0\end{array}$

Figure S2: 1H-NMR and 13C-NMR of compound 3 


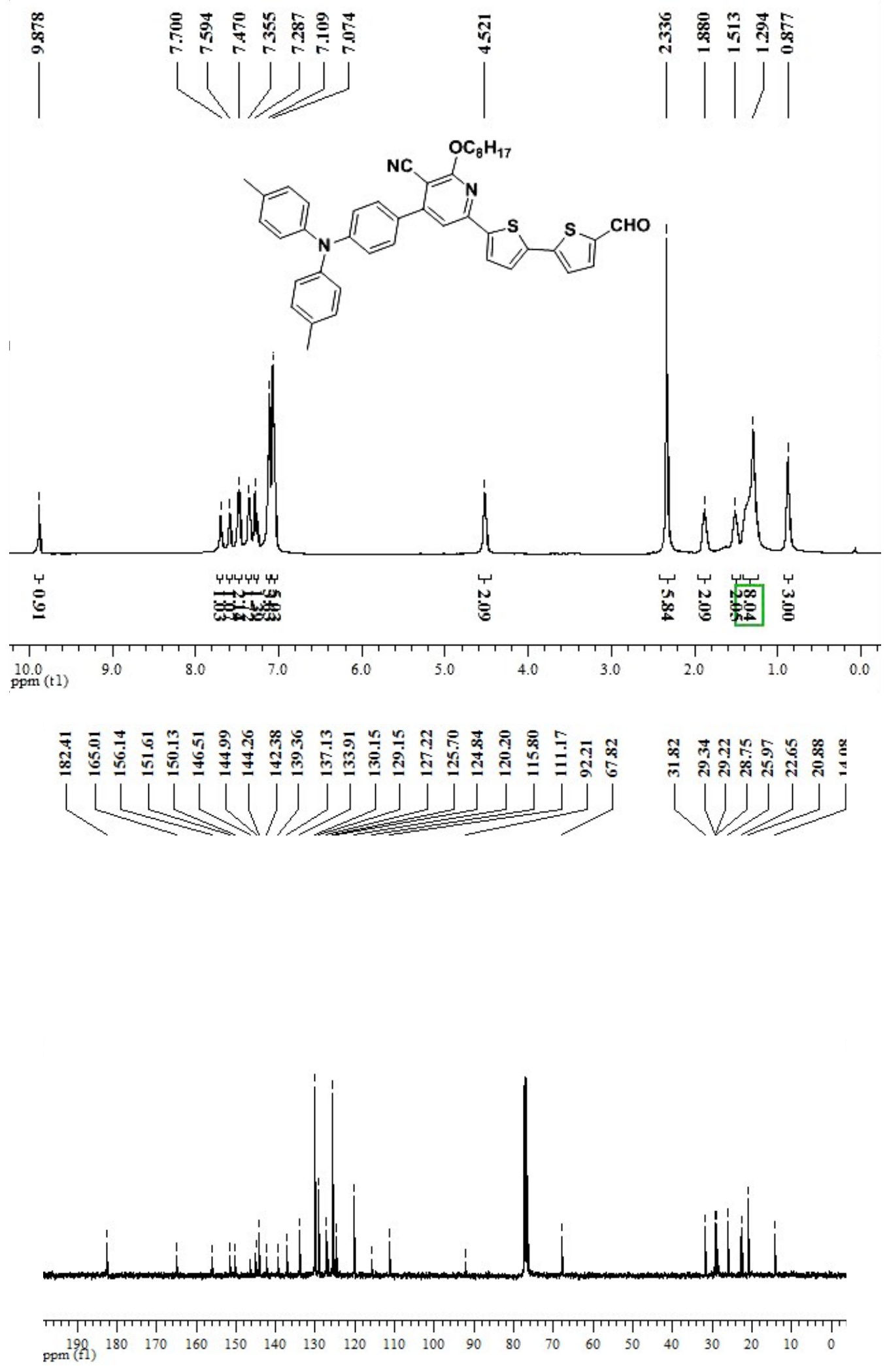

Figure S3: 1H-NMR and 13C-NMR of compound 4 

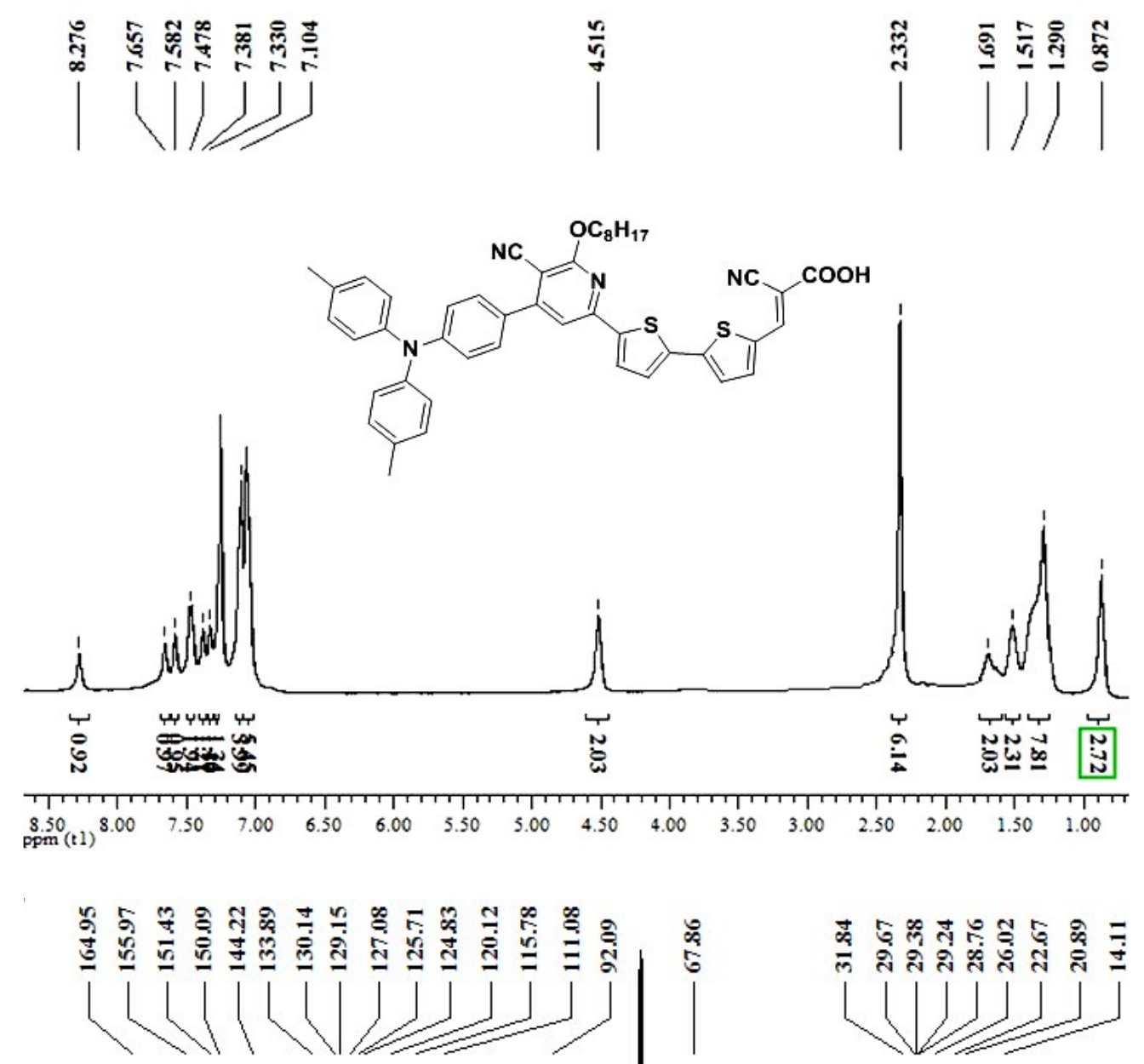

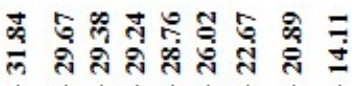

\section{:}

(4)

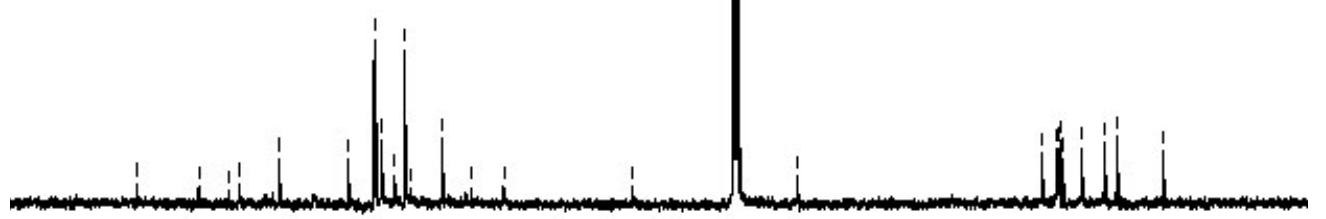

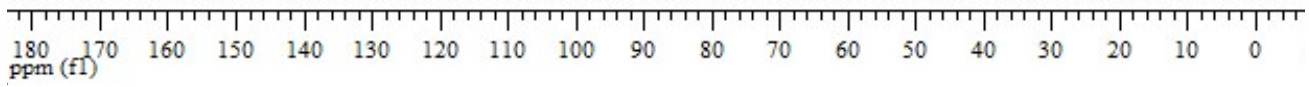

Figure S4: 1H-NMR and 13C-NMR of TPCTh 

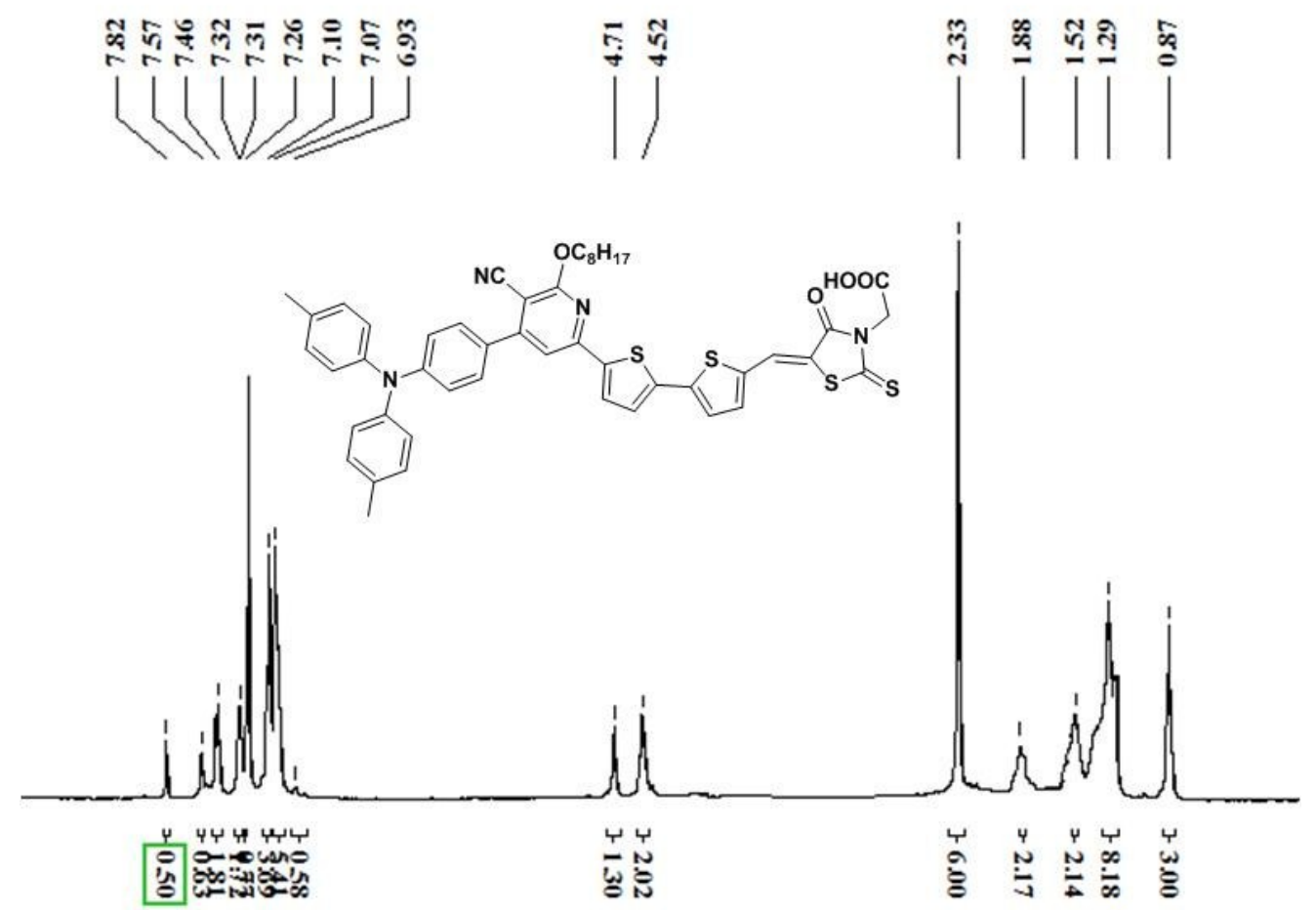

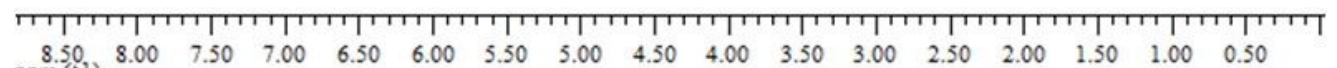

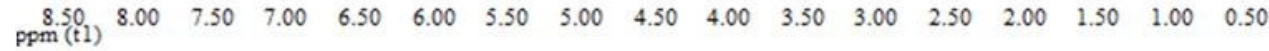

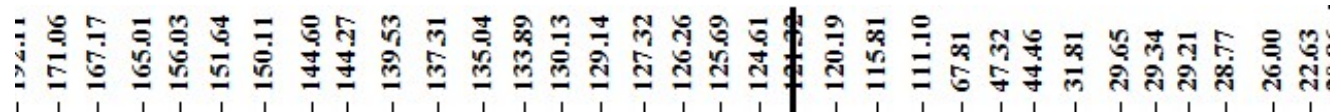
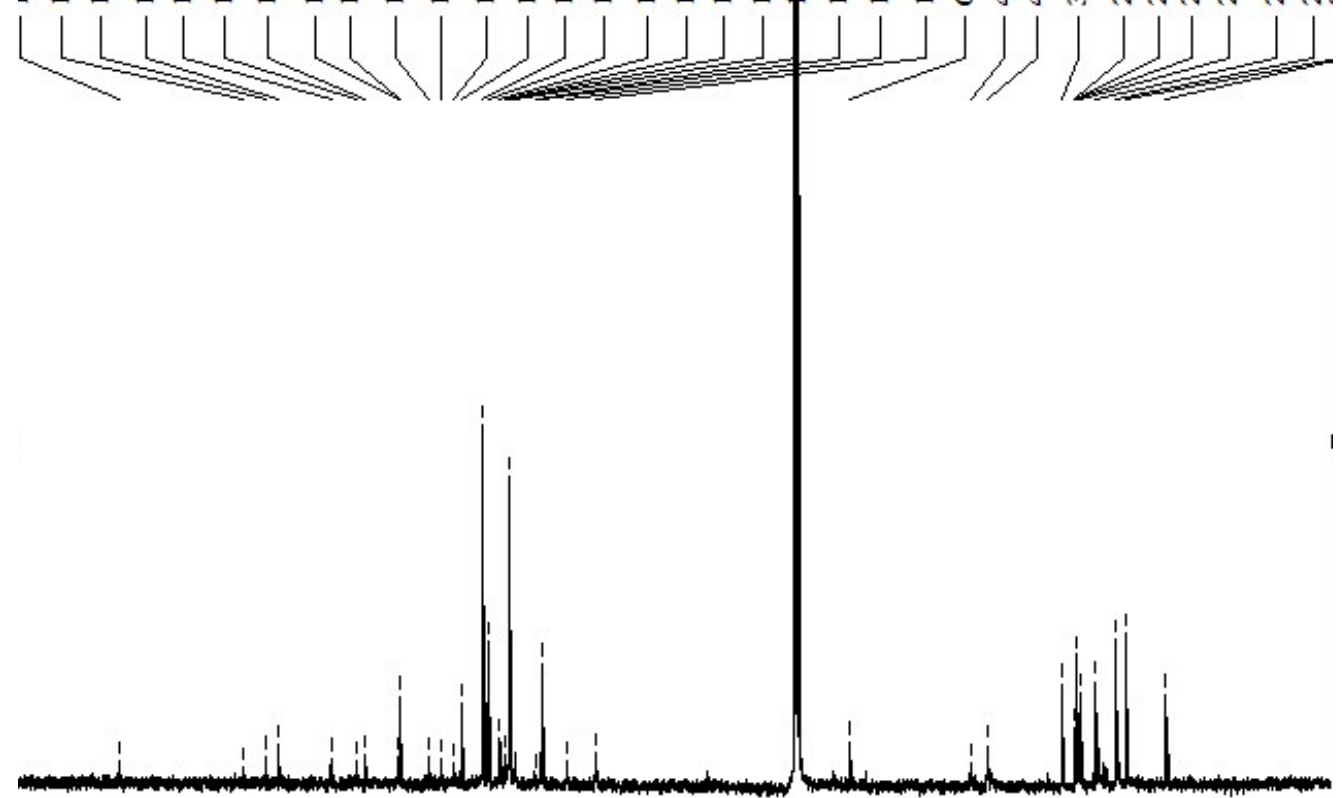

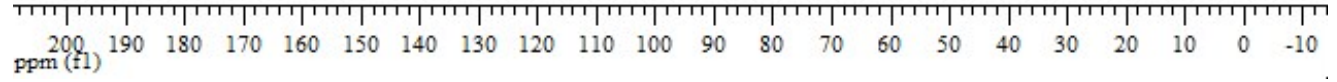

Figure S5: 1H-NMR and 13C-NMR of TPCRh 


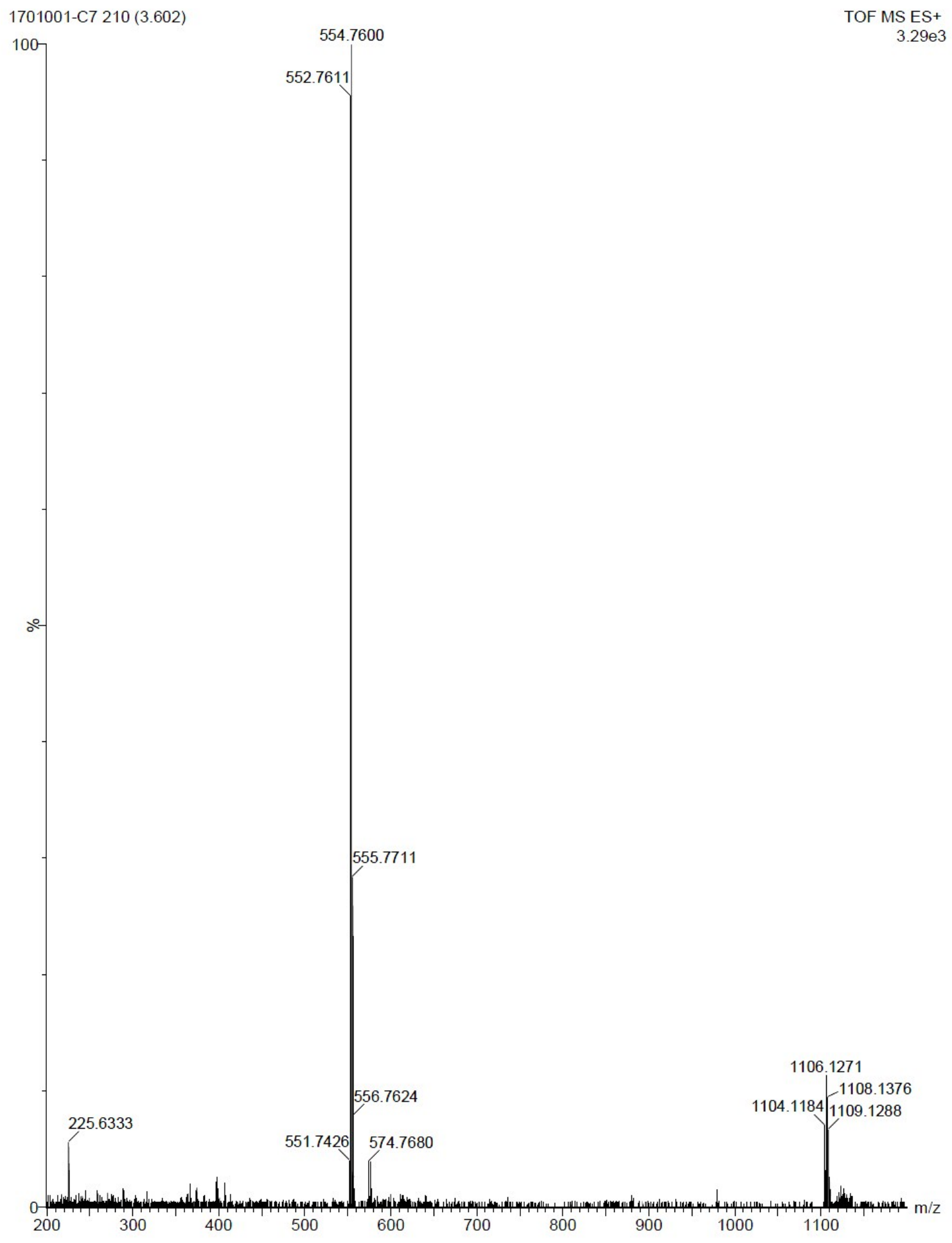

Figure S6: Mass spectrum of compound 2 


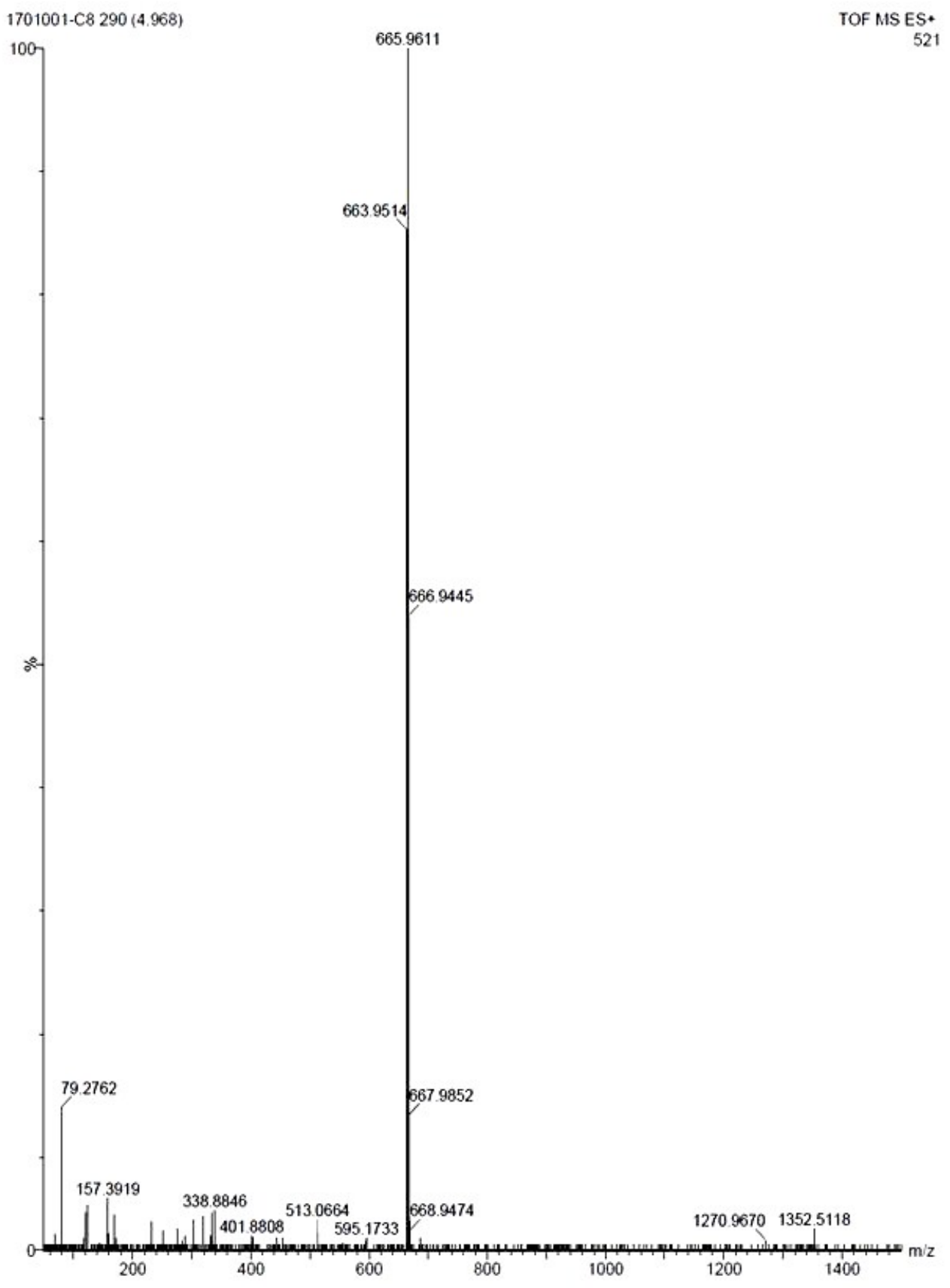

Figure S7: Mass spectrum of compound 3 


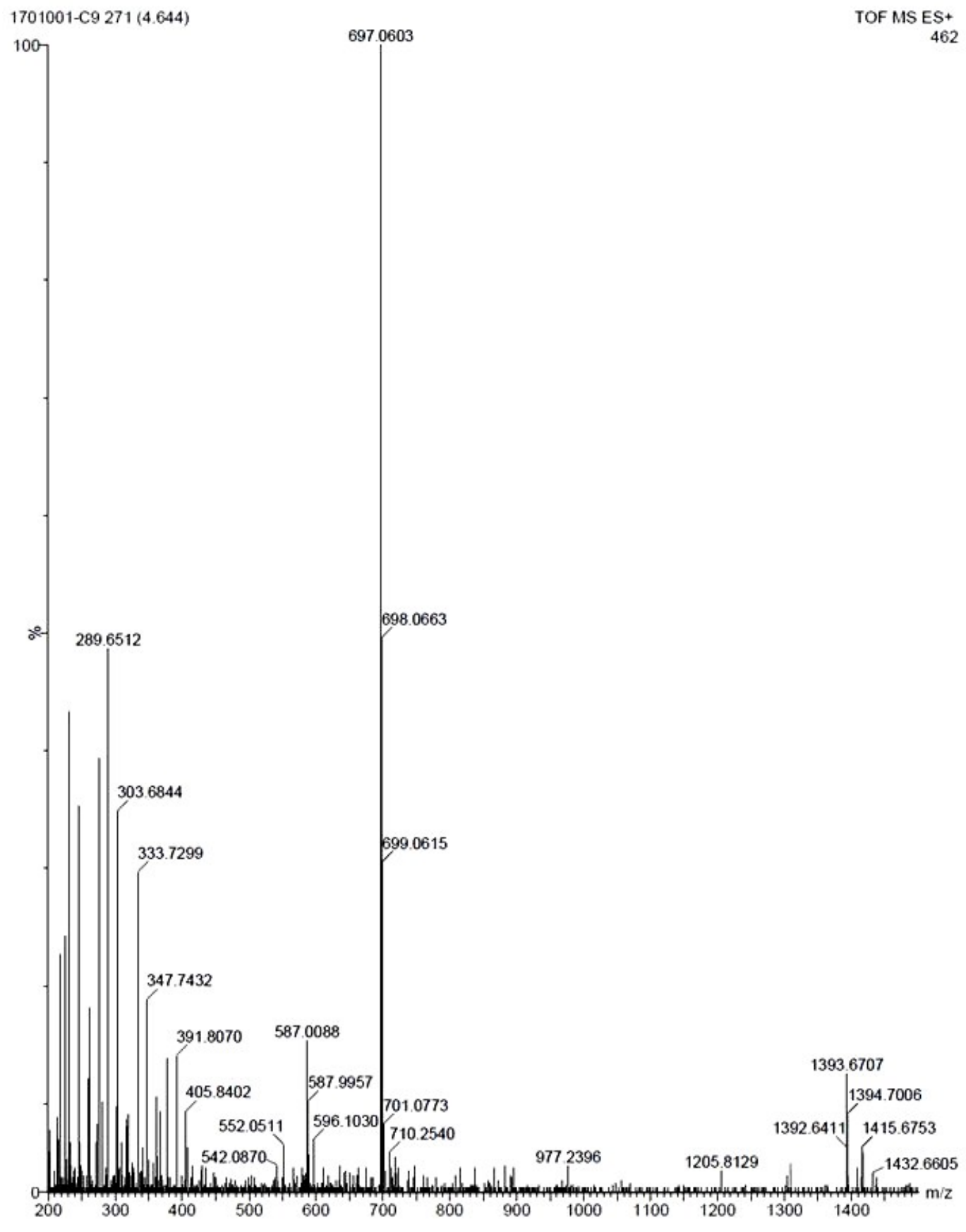

Figure S8: Mass spectrum of compound 4 


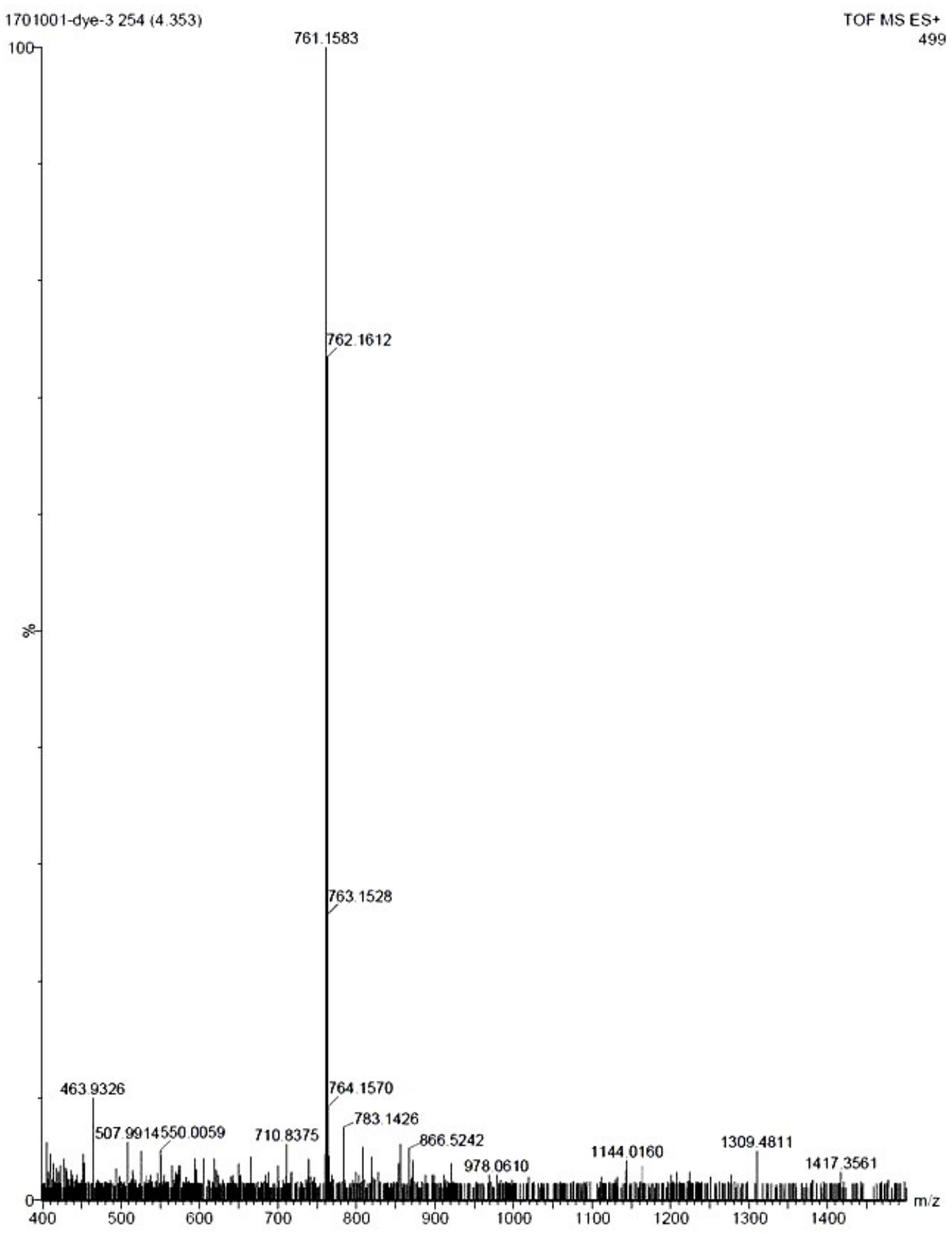

Figure S9: Mass spectrum of TPCTh 


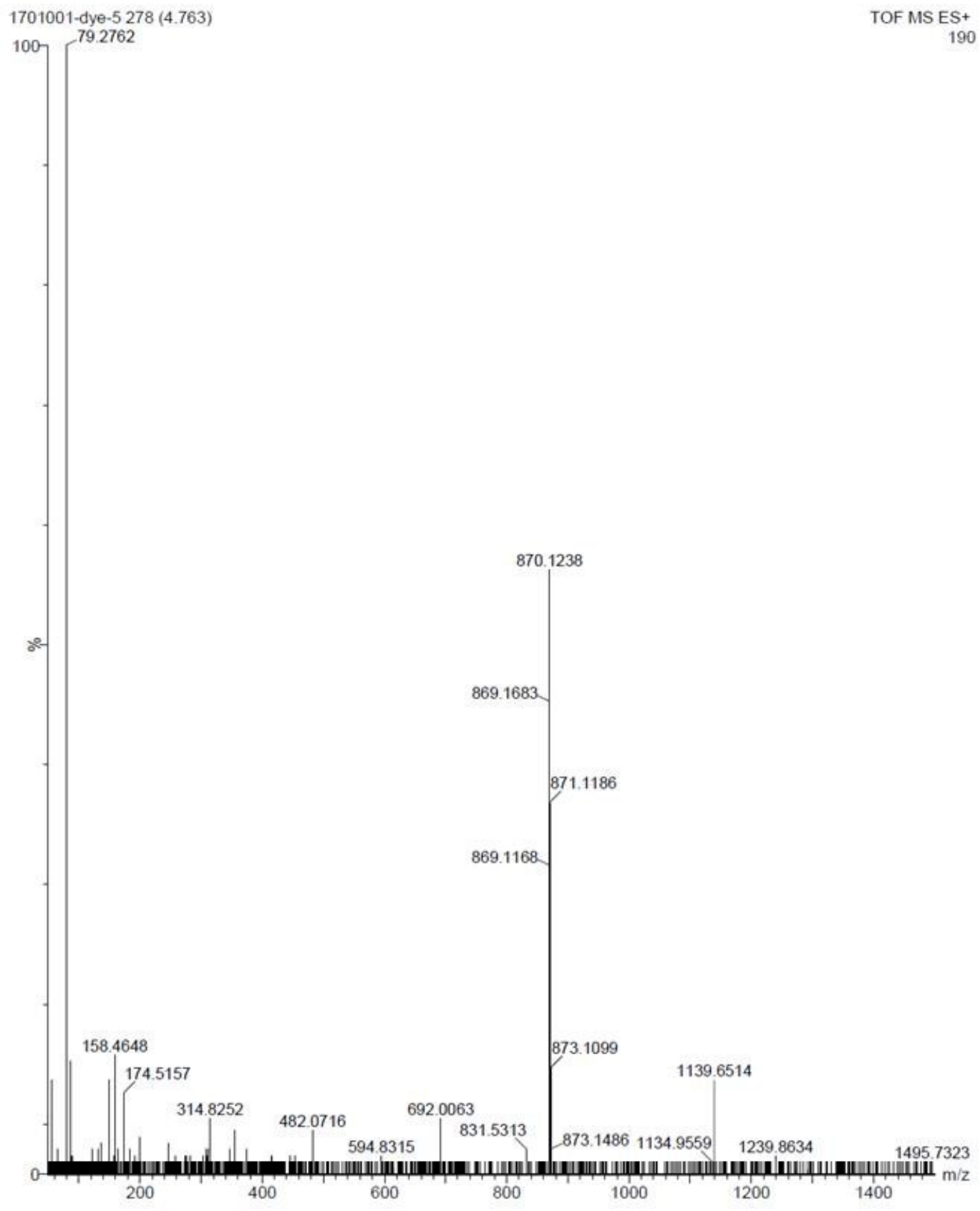

Figure S10: Mass spectrum of TPCRh 


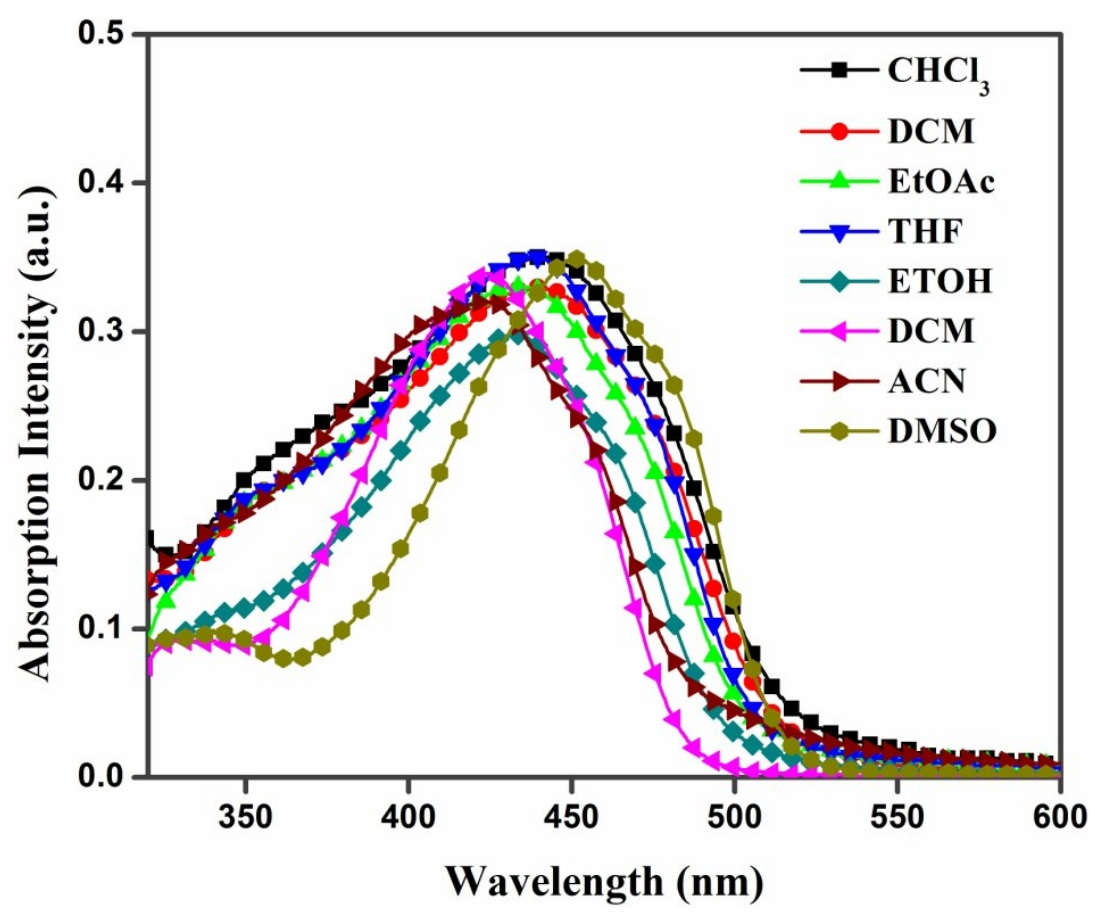

Figure S11: Solvatochromism of TPCTh

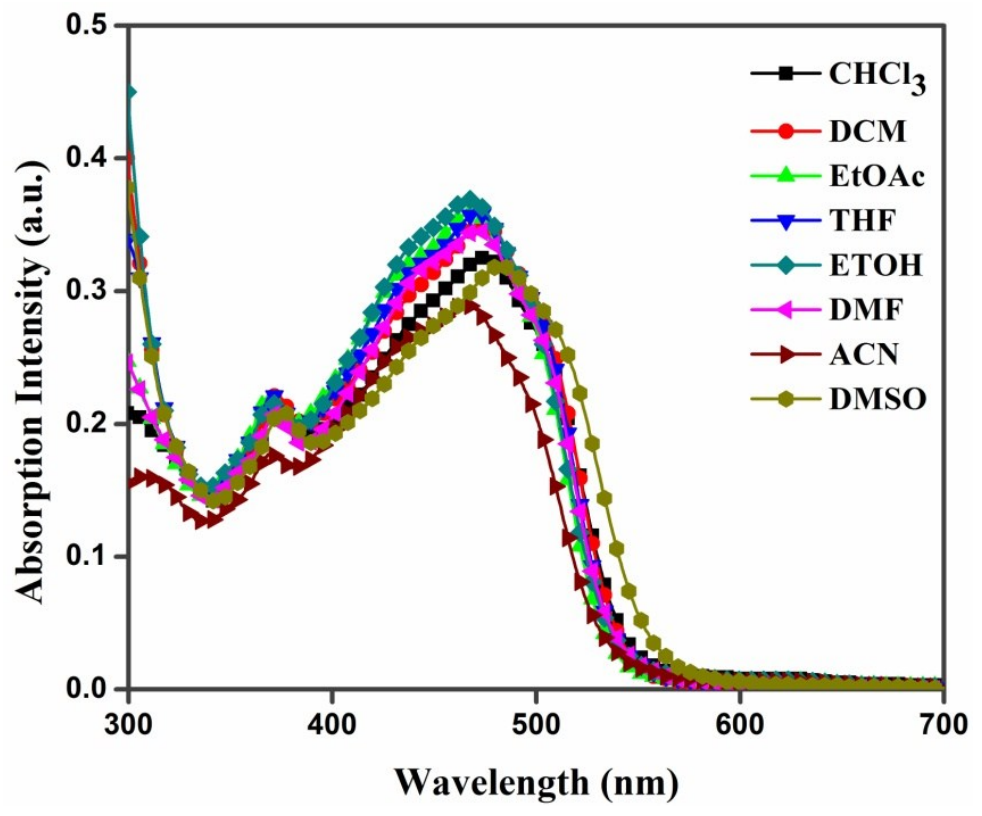

Figure S12: Solvatochromism of TPCRh 


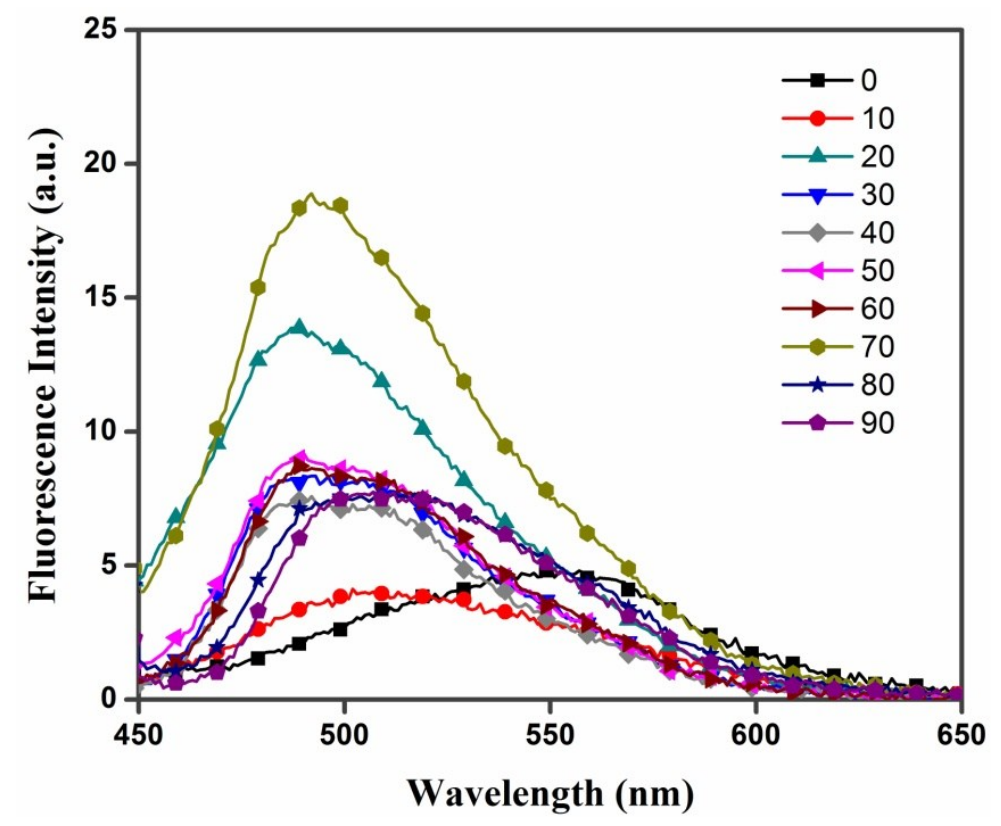

Figure S13: Aggregation study of TPCTh

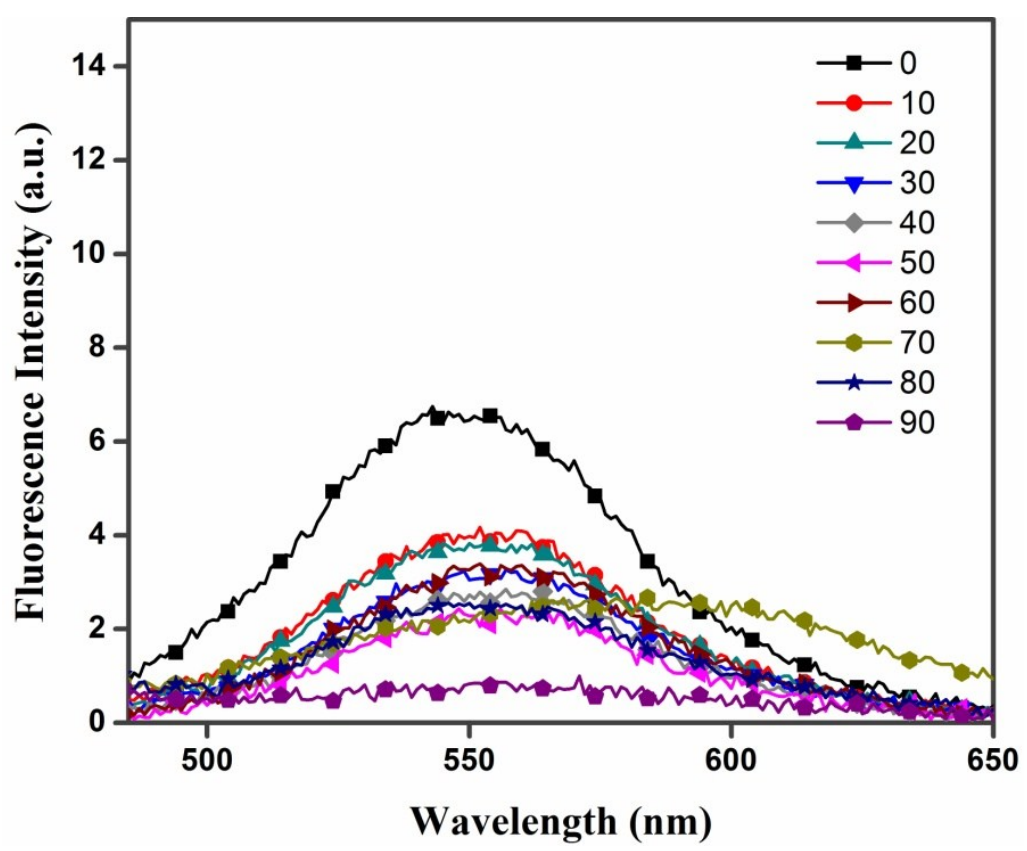

Figure S14: Aggregation of TPCRh 


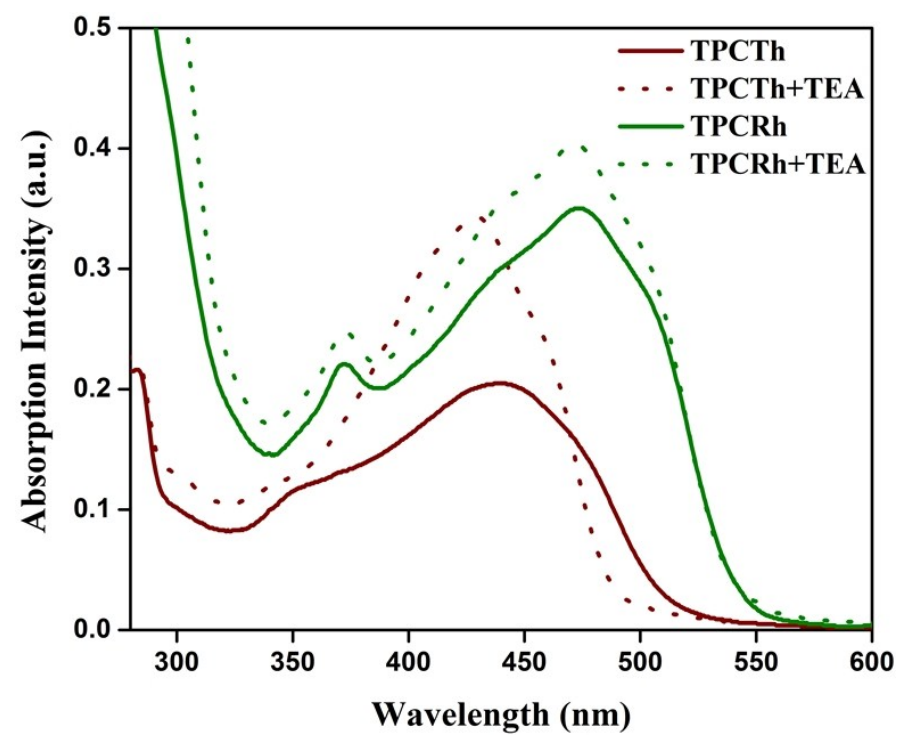

Figure S15: Absorption spectra of dyes in presence TEA.

Table S1: Cartesian coordinates for the optimized geometry of TPCTh

\begin{tabular}{lrrr}
\multicolumn{4}{l}{ Energy: -1721661.3463998} \\
$\mathrm{C}$ & -5.32740 & 0.15623 & -0.04325 \\
$\mathrm{C}$ & -4.97806 & -1.01978 & -0.73519 \\
$\mathrm{C}$ & -4.32795 & 0.80278 & 0.71019 \\
$\mathrm{C}$ & -3.03971 & 0.29017 & 0.76227 \\
$\mathrm{C}$ & -2.69029 & -0.89591 & 0.09141 \\
$\mathrm{C}$ & -3.69290 & -1.53663 & -0.65780 \\
$\mathrm{C}$ & -1.30747 & -1.41218 & 0.16140 \\
$\mathrm{C}$ & -0.22629 & -0.51815 & 0.09624 \\
$\mathrm{C}$ & 1.08573 & -0.99106 & 0.15833 \\
$\mathrm{~N}$ & 1.37305 & -2.30778 & 0.27540 \\
$\mathrm{C}$ & 0.36948 & -3.16375 & 0.34118 \\
$\mathrm{C}$ & -1.00143 & -2.78528 & 0.29852 \\
$\mathrm{C}$ & 2.23836 & -0.09981 & 0.08808 \\
$\mathrm{C}$ & -1.99275 & -3.80018 & 0.45032 \\
$\mathrm{C}$ & 2.29382 & 1.27627 & -0.02500
\end{tabular}




\begin{tabular}{|c|c|c|c|}
\hline $\mathrm{C}$ & 3.60930 & 1.78431 & -0.06544 \\
\hline $\mathrm{C}$ & 4.58022 & 0.79883 & 0.01981 \\
\hline $\mathrm{C}$ & 3.84472 & -0.78364 & 0.15617 \\
\hline 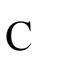 & 6.01175 & .98913 & 0.00874 \\
\hline$c$ & 6.70143 & 2.18291 & 0.20048 \\
\hline $\mathrm{C}$ & 8.09652 & 2.05217 & 0.12831 \\
\hline $\mathrm{C}$ & 8.52118 & 0.74882 & -0.11734 \\
\hline S & 7.12822 & -0.31743 & -0.27192 \\
\hline $\mathrm{C}$ & 9.82290 & 0.18135 & -0.25603 \\
\hline $\mathrm{C}$ & 11.05084 & 0.78436 & -0.19168 \\
\hline $\mathrm{C}$ & 12.24536 & -0.08093 & -0.37842 \\
\hline $\mathrm{C}$ & 11.23166 & 2.17768 & 0.04239 \\
\hline $\mathrm{N}$ & -6.63679 & 0.66608 & -0.09940 \\
\hline $\mathrm{C}$ & -7.75472 & -0.20994 & -0.24772 \\
\hline $\mathrm{C}$ & -6.87859 & 2.06676 & 0.02308 \\
\hline $\mathrm{C}$ & -7.87473 & 2.54283 & 0.88597 \\
\hline $\mathrm{C}$ & -8.12594 & 3.90932 & 0.98382 \\
\hline $\mathrm{C}$ & -7.38772 & 4.84404 & 0.24651 \\
\hline $\mathrm{C}$ & -6.39258 & 4.35551 & -0.61107 \\
\hline $\mathrm{C}$ & -6.14318 & 2.99103 & -0.73300 \\
\hline $\mathrm{C}$ & -7.86233 & -1.37567 & 0.52378 \\
\hline $\mathrm{C}$ & -8.95780 & -2.22103 & 0.36880 \\
\hline $\mathrm{C}$ & -9.98833 & -1.92599 & -0.53396 \\
\hline $\mathrm{C}$ & -9.87303 & -0.75316 & -1.29126 \\
\hline $\mathrm{C}$ & -8.77352 & 0.09190 & -1.16119 \\
\hline $\mathrm{C}$ & -11.19273 & -2.82718 & -0.66515 \\
\hline $\mathrm{C}$ & -7.63840 & 6.32644 & 0.38709 \\
\hline $\mathrm{N}$ & -2.78778 & -4.64076 & 0.58121 \\
\hline $\mathrm{N}$ & 11.34245 & 3.32136 & 0.23572 \\
\hline $\mathrm{O}$ & 13.39943 & 0.61539 & -0.29326 \\
\hline $\mathrm{O}$ & 12.20551 & -1.27798 & -0.58452 \\
\hline
\end{tabular}




\begin{tabular}{|c|c|c|c|}
\hline $\mathrm{O}$ & 0.61920 & -4.47514 & 0.46592 \\
\hline$C$ & 1.99036 & -4.88904 & 0.53726 \\
\hline & 9.87656 & -0.88955 & -0.44223 \\
\hline $\mathrm{H}$ & -5.72194 & -1.52580 & -1.33934 \\
\hline $\mathrm{H}$ & -4.57316 & 1.69920 & 1.26774 \\
\hline $\mathrm{H}$ & -2.30071 & 0.79480 & 1.37762 \\
\hline $\mathrm{H}$ & -3.46070 & -2.43660 & -1.21561 \\
\hline $\mathrm{H}$ & -0.42237 & 0.53790 & -0.03800 \\
\hline $\mathrm{H}$ & 1.41336 & 1.90482 & -0.08444 \\
\hline $\mathrm{H}$ & 3.84193 & 2.83677 & -0.17549 \\
\hline $\mathrm{H}$ & 6.20014 & 3.12014 & 0.40939 \\
\hline $\mathrm{H}$ & 8.78319 & 2.87795 & 0.26076 \\
\hline $\mathrm{H}$ & -8.45398 & 1.83723 & 1.47231 \\
\hline $\mathrm{H}$ & -8.90676 & 4.25568 & 1.65628 \\
\hline П & -5.81301 & 5.05402 & -1.20974 \\
\hline $\mathrm{H}$ & -5.38078 & 2.63451 & -1.41806 \\
\hline $\mathrm{H}$ & -7.08246 & -1.62114 & 1.23706 \\
\hline $\mathrm{H}$ & -9.01667 & -3.12331 & 0.97222 \\
\hline $\mathrm{H}$ & -10.65079 & -0.50150 & -2.00801 \\
\hline $\mathrm{H}$ & -8.69860 & 0.98892 & -1.76712 \\
\hline $\mathrm{H}$ & -11.96601 & -2.56865 & 0.06935 \\
\hline $\mathrm{H}$ & -11.64740 & -2.74552 & -1.65689 \\
\hline $\mathrm{H}$ & -10.92807 & -3.87590 & -0.49988 \\
\hline $\mathrm{H}$ & -6.98417 & 6.77158 & 1.14744 \\
\hline $\mathrm{H}$ & -7.44873 & 6.85577 & -0.55173 \\
\hline 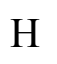 & -8.67018 & 6.53111 & 0.68741 \\
\hline $\mathrm{H}$ & 14.11579 & -0.02730 & -0.42657 \\
\hline $\mathrm{H}$ & 2.48952 & -4.43756 & 1.39837 \\
\hline $\mathrm{H}$ & 1.95306 & -5.97303 & 0.64231 \\
\hline $\mathrm{H}$ & 2.52973 & -4.61064 & -0.37158 \\
\hline
\end{tabular}


Table S2: Cartesian coordinates for the optimized geometry of TPCRh

Energy: -2317467.9254681

$\begin{array}{llll}\mathrm{C} & -5.93997 & -0.96129 & -0.01491 \\ \mathrm{C} & -6.32177 & 0.11472 & 0.80972 \\ \mathrm{C} & -4.79416 & -0.80434 & -0.81947 \\ \mathrm{C} & -4.06744 & 0.37693 & -0.79160 \\ \mathrm{C} & -4.45526 & 1.46286 & 0.01453 \\ \mathrm{C} & -5.60122 & 1.30044 & 0.81290 \\ \mathrm{C} & -3.65250 & 2.70302 & 0.02823 \\ \mathrm{C} & -2.25010 & 2.62489 & -0.00218 \\ \mathrm{C} & -1.47238 & 3.78366 & 0.00731 \\ \mathrm{~N} & -2.02235 & 5.02014 & 0.05738 \\ \mathrm{C} & -3.33817 & 5.11209 & 0.08762 \\ \mathrm{C} & -4.22107 & 3.99548 & 0.06510 \\ \mathrm{C} & -0.01111 & 3.75067 & -0.02302 \\ \mathrm{C} & -5.62644 & 4.23872 & 0.02696 \\ \mathrm{C} & 0.84292 & 4.83392 & 0.02146 \\ \mathrm{C} & 2.20890 & 4.48116 & -0.02122 \\ \mathrm{C} & 2.42539 & 3.11624 & -0.10482 \\ \mathrm{~S} & 0.89759 & 2.25922 & -0.13701 \\ \mathrm{C} & 3.69408 & 2.42963 & -0.17136 \\ \mathrm{C} & 4.92997 & 2.97858 & -0.48888 \\ \mathrm{C} & 5.98615 & 2.05059 & -0.46494 \\ \mathrm{C} & 5.59012 & 0.75969 & -0.13343 \\ \mathrm{~S} & 3.85793 & 0.72633 & 0.16894 \\ \mathrm{C} & 6.32980 & -0.45706 & -0.00779 \\ \mathrm{C} & 7.66087 & -0.67828 & -0.17477 \\ \mathrm{~N} & -6.68133 & -2.15601 & -0.03767 \\ \mathrm{C} & -6.04443 & -3.40153 & -0.31992 \\ & -8.08748 & -2.15013 & 0.20889 \\ & & -3.77281 & 0.34432\end{array}$




\begin{tabular}{|c|c|c|c|}
\hline $\mathrm{C}$ & -4.25867 & -4.99440 & 0.06604 \\
\hline $\mathrm{C}$ & -4.80667 & -5.89016 & -0.86167 \\
\hline $\mathrm{C}$ & -5.99236 & -5.51386 & -1.50669 \\
\hline$C$ & -6.60135 & -4.28774 & -1.25188 \\
\hline 4 & -8.65853 & -3.09812 & 1.06805 \\
\hline $\mathrm{C}$ & -10.03351 & -3.10584 & 1.29186 \\
\hline $\mathrm{C}$ & -10.87874 & -2.16806 & 0.68576 \\
\hline $\mathrm{C}$ & -10.29355 & -1.21873 & -0.16357 \\
\hline $\mathrm{C}$ & -8.92388 & -1.21123 & -0.41232 \\
\hline $\mathrm{C}$ & -4.13092 & -7.20411 & -1.17317 \\
\hline $\mathrm{C}$ & -12.37068 & -2.19180 & 0.91710 \\
\hline S & 8.94172 & 0.45588 & -0.60580 \\
\hline $\mathrm{C}$ & 10.19046 & -0.80758 & -0.54825 \\
\hline $\mathrm{N}$ & 9.61056 & -2.01149 & -0.23244 \\
\hline $\mathrm{C}$ & 8.21793 & -2.03098 & 0.00742 \\
\hline $\mathrm{O}$ & 7.61426 & -3.04822 & 0.29720 \\
\hline $\mathrm{C}$ & 10.38315 & -3.21287 & -0.00839 \\
\hline $\mathrm{C}$ & 10.91747 & -3.27092 & 1.43085 \\
\hline S & 11.78198 & -0.52050 & -0.85877 \\
\hline $\mathrm{N}$ & -6.76973 & 4.45661 & -0.00941 \\
\hline $\mathrm{O}$ & 11.76550 & -4.29918 & 1.67131 \\
\hline $\mathrm{O}$ & 10.60075 & -2.50290 & 2.29721 \\
\hline $\mathrm{O}$ & -3.92037 & 6.32147 & 0.13179 \\
\hline $\mathrm{C}$ & -3.06626 & 7.47102 & 0.13269 \\
\hline $\mathrm{H}$ & 5.77271 & -1.35267 & 0.26188 \\
\hline $\mathrm{H}$ & -7.18711 & 0.01408 & 1.45418 \\
\hline $\mathrm{H}$ & -4.48695 & -1.60908 & -1.47719 \\
\hline $\mathrm{H}$ & -3.20604 & 0.47610 & -1.44539 \\
\hline $\mathrm{H}$ & -5.91998 & 2.09933 & 1.47252 \\
\hline $\mathrm{H}$ & -1.77891 & 1.64912 & 0.00349 \\
\hline $\mathrm{H}$ & 0.46906 & 5.84673 & 0.09325 \\
\hline
\end{tabular}




\begin{tabular}{|c|c|c|c|}
\hline & 3.01704 & 5.20099 & 0.03222 \\
\hline & 5.05571 & 4.02125 & -0.75411 \\
\hline $\mathrm{H}$ & 7.00886 & 2.32036 & -0.69670 \\
\hline & -4.43487 & -3.10284 & 1.08053 \\
\hline & -3.34782 & -5.26386 & 0.59495 \\
\hline & -6.44409 & -6.18776 & -2.23038 \\
\hline & -7.51570 & -4.01296 & -1.76741 \\
\hline & -8.02127 & -3.82617 & 1.55911 \\
\hline & -10.45501 & -3.84889 & 1.96424 \\
\hline & -10.92149 & -0.47697 & -0.65073 \\
\hline & -8.49676 & -0.47256 & -1.08251 \\
\hline $\mathrm{H}$ & -3.43757 & -7.10571 & -2.01808 \\
\hline 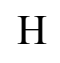 & -3.55150 & -7.56844 & -0.31965 \\
\hline & -4.85912 & -7.97554 & -1.44119 \\
\hline & -12.89169 & -2.71773 & 0.10709 \\
\hline & -12.62228 & -2.70229 & 1.85120 \\
\hline 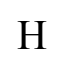 & -12.78526 & -1.17998 & 0.96311 \\
\hline & 9.71340 & -4.06318 & -0.17029 \\
\hline & 11.20870 & -3.24254 & -0.72476 \\
\hline & 11.94363 & -4.80075 & 0.86208 \\
\hline & -2.41216 & 7.47123 & 1.00866 \\
\hline & -2.45015 & 7.50095 & -0.76989 \\
\hline & -3.74151 & 8.32600 & 0.16175 \\
\hline
\end{tabular}

Table S3: Lifetime measured for CCTh, TPCTh and TPCRh as sensitizers.

\begin{tabular}{ccc}
\hline Dye & $\begin{array}{c}\text { Frequency Max } \\
(\mathbf{H z})\end{array}$ & $\begin{array}{c}\text { Lifetime } \\
(\mathbf{S})\end{array}$ \\
\hline CCTh & 83.77 & $1.90 \times 10^{-3}$ \\
\hline TPCTh & 119.38 & $1.33 \times 10^{-3}$ \\
\hline TPCRh & 492.39 & $3.23 \times 10^{-4}$ \\
\hline
\end{tabular}




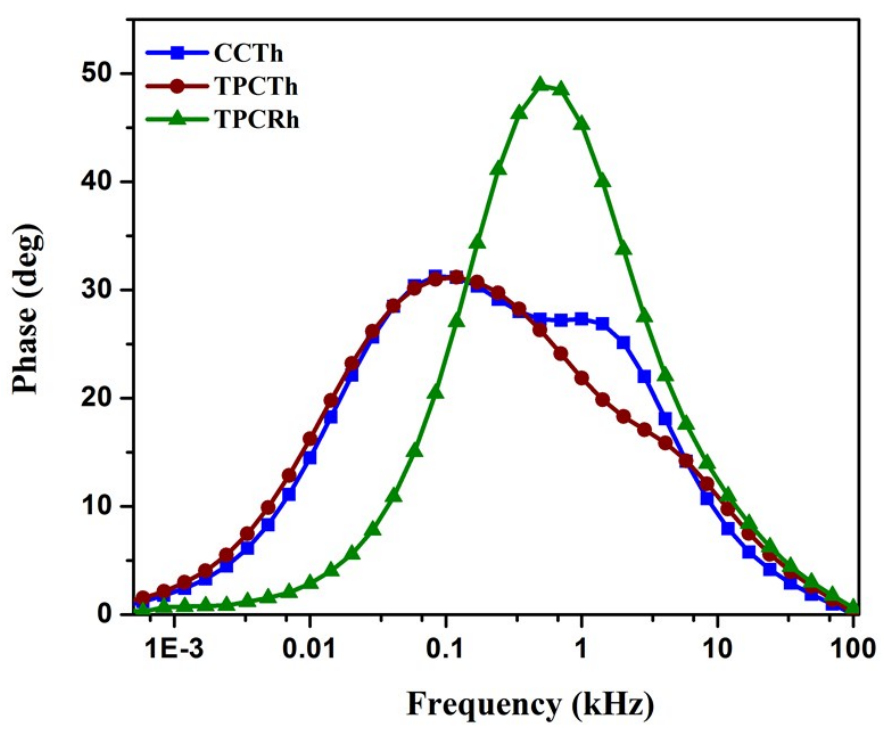

Figure S16. Bode plots of DSSCs fabricated with CCTh, TPCTh and TPCRh as sensitizers at $0.60 \mathrm{~V}$ under forward bias in dark.

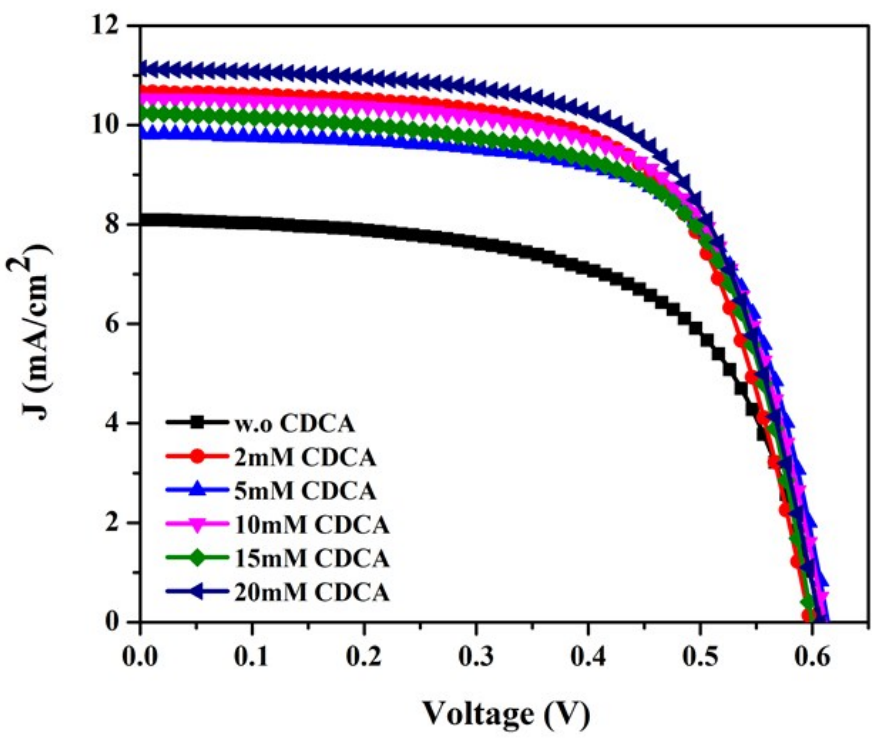

Figure S17. DSSCs fabricated with CDCA with dye TPCTh. 
Table S4: DSSC device parameters of TPCTh fabricated with CDCA.

\begin{tabular}{lllll}
\hline & Voc $(\mathbf{m V})$ & Jsc $\left(\mathbf{m A c m}^{-2}\right)$ & FF (\%) & Efficiency (\%) \\
\hline w.o CDCA & 609 & 8.1 & 60.78 & 2.99 \\
\hline 2mM CDCA & 598 & 10.67 & 64.56 & 4.12 \\
\hline $5 \mathrm{mM} \mathrm{CDCA}$ & 614 & 9.81 & 67.11 & 4.04 \\
\hline $10 \mathrm{mM} \mathrm{CDCA}$ & 611 & 10.55 & 64.67 & 4.17 \\
\hline $15 \mathrm{mM} \mathrm{CDCA}$ & 599 & 10.25 & 65.4 & 4.02 \\
\hline 20mM CDCA & 607 & 11.14 & 64.56 & 4.36 \\
\hline
\end{tabular}

Table S5: Dye loading.

\begin{tabular}{cc}
\hline Dye & $\begin{array}{c}\text { Dye-loading } \\
\left(\mathbf{X 1 0}^{-7}\right) \mathbf{M o l} \mathbf{~ c m}^{-2}\end{array}$ \\
\hline CCTh & 1.92 \\
\hline TPCTh & 0.92 \\
\hline TPCRh & 0.96 \\
\hline
\end{tabular}

\section{Reference}

1. A. Zaban, M. Greenshtein and J. Bisquert, ChemPhysChem, 2003, 4, 859-864.

2. J. Krüger, R. Plass, M. Grätzel, P. J. Cameron and L. M. Peter, The Journal of Physical Chemistry B, 2003, 107, 7536-7539.

3. L. M. Peter, N. W. Duffy, R. L. Wang and K. G. U. Wijayantha, Journal of Electroanalytical Chemistry, 2002, 524-525, 127-136.

4. S. Soman, M. A. Rahim, S. Lingamoorthy, C. H. Suresh and S. Das, Physical Chemistry Chemical Physics, 2015, 17, 23095-23103.

5. G. Gokul, S. C. Pradhan and S. Soman, 2019, DOI: 10.1007/978-981-13-3302-6_9, pp. 281-316.

6. J. S Panicker, B. Balan, S. Soman and V. Nair, Understanding structure-property correlation of metal free organic dyes using interfacial electron transfer measurements, 2016. 
$* * * * * * * * * * *$ 